\title{
NON-LANGERHANS CELLS HISTIOCYTOSIS ASSOCIATED WITH SYSTEMIC LUPUS ERYTHEMATOSUS - CASE REPORT
}

Izaura Tereza Silva Guedes ${ }^{1, \star}$, Edgard Torres dos Reis Neto ${ }^{1}$, Renan Rodrigues Neves Ribeiro do Nascimento ${ }^{1}$, Isaac Felipe Leite Braz ${ }^{1}$, Miriam Fang Castro ${ }^{1}$, Rodrigo Lorenzetti Serrano ${ }^{1}$, Guilherme Pereira Carlesso ${ }^{1}$, Felipe de Lacerda Veiga ${ }^{1}$, Lucas de Castro Barroti ${ }^{1}$, Leonardo Afonso Costa ${ }^{1}$, João Paulo Peres Lima ${ }^{1}$

1.Universidade Federal de São Paulo, São Paulo (SP), Brazil.

*Corresponding author: terezaguedes.i@gmail.com

\section{BACKGROUND}

Histiocytosis refers to a group of idiopathic and rare diseases, resulting from tissue infiltration and abnormal behavior of histiocytes, an archaic name given to cells residing in tissues, such as macrophages, Langerhans cells and other dendritic cells. The clinical presentation of histiocytosis varies depending on the location and extent of tissue involvement. The definitive diagnosis is challenging, based on clinical findings, imaging tests and biopsy with immunohistochemical evaluation. Non-Langerhans histiocytosis (such as Rosai-Dorfman disease, juvenile xanthogranuloma, and Erdheim-Chester disease) are characterized by the absence of Langerhans cell markers, such as Langerin and Birbeck granules, being rarely associated with autoimmune diseases.

\section{CASE REPORT}

Female patient, 45 years old, Caucasian, with no previous comorbidities, hospitalized due to pleural and pericardial effusion, associated with wasting syndrome, generalized lymph node enlargement, xanthelasma and daily afternoon fever for 2 months. Infectious causes were ruled out and axillary lymph node biopsy was performed as an additional diagnostic tool. During hospitalization, dysmorphic hematuria, alopecia and arthralgia were identified, associated with ANA in high titers with positive anti-DNA, anti-nucleosome and anti-Ro, besides complement consumption. The biopsy result showed intense lymphoid hyperplasia with positive CD68, negative CD1a and negative $\mathrm{S100}$, suggesting non-Langerhans cell lymphohistiocytosis. The Kikuchi lymphadenitis hypothesis was ruled out by the negativity for myeloperoxidase in the histiocytic population. Treatment was performed with glucocorticoid in pulse therapy and hydroxychloroquine, with descent of fever and resolution of serositis.

\section{CONCLUSION}

The association of non-Langerhans cell lymphohistiocytosis with autoimmune rheumatic diseases is rare, not being frequently found in other case reports. The overlap of systemic lupus erythematosus with non-Langerhans cell lymphohistiocytosis makes the treatment challenging. Glucocorticoids were chosen as a therapeutic strategy due to the good results in patients with lupus, leading to the reversal of part of the symptoms. The case in question could still be better clarified based on the search for mutations related to the different subtypes of lymphohistiocytosis, providing a more accurate diagnosis and, consequently, better handling of the case.

\section{KEYWORDS}

Histiocytosis, Non-Langerhans histiocytosis, Erdheim-Chester, Systemic lupus erythematosus. 